\title{
PSEUDOTUMOR CEREBRI
}

\section{VITAMIN A AND IDIOPATHIC INTRACRANIAL HYPERTENSION}

Vitamin A levels were measured in the cerebrospinal fluid of a total of 78 patients having idiopathic intracranial hypertension ( IIH; $\mathrm{n}=20$ ), elevated pressure of other causes $(E-I C P ; n=19)$, and normal pressure $(N-I C P ; n=39)$, in a study at the University of Utah, Salt Lake City, UT. Higher CSF vitamin A levels found in some patients with IIH were significantly correlated $(p=0.036)$ when compared with control E-ICP and IIH patients. Higher levels were marginally significantly associated with female gender $(\mathrm{p}=0.094)$ and younger age $(\mathrm{p}=0.069)$. Multivitamin use was not associated with the levels of vitamin A. High vitamin A levels in patients with IIH and high average vitamin A levels in patients with breakdown of the blood-brain barrier are suggestive of a toxic effect of CSF retinol on the arachnoid granulation resorption mechanism in IIH. (Warner JEA, Bernstein PS, Yemelyanov A et al. Vitamin A in the cerebrospinai fiuid of patients with and without idiopathic intracranial hypertension. Ann Neurol November 2002;52:647-650). (Respond: Dr Warner, Department of Ophthalmology and Visual Sciences, John A Moran Eye Center, 50 North Medical Drive, Salt Lake City, UT 84132).

COMMENT. Some patients with idiopathic intracranial hypertension have significantly higher levels of vitamin A in the CSF than patients with normal pressure or those with increased pressure due to other causes. Serum retinolbinding protein (RBP) and retinol are also elevated in some patients with IIH (Jacobson DM, Berg R, Wall M, et al. Neurology 1999;53:1114-1118). Vitamin A may have a specific transport mechanism into the CSF, and it becomes toxic when the level exceeds the RBP binding capacity. Study of patients with breakdown of the blood-brain barrier may elucidate the transport mechanism of CSF vitamin A and pathogenesis of IIH.

The role of vitamin A intoxication in the pathogenesis of IIH is discussed in an editorial by Fishman RA. (Polar bear liver, vitamin A, aquaporins, and pseudotumor cerebri. Ann Neurol November 2002;52:531-533). It is speculated that vitamin $\mathrm{A}$ increases CSF volume and pressure by an effect on aquaporins present in membranes of the choroid plexus that control CSF secretion.

\section{ATTENTION DEFICIT DISORDERS}

\section{BRAIN VOLUME ABNORMALITIES IN ADHD}

Regional brain volumes have been compared at initial MRI scans and their change over time in 152 medicated and previously unmedicated male and female patients (age range, 5-18 years) with attention-deficit/hyperactivity disorder (ADHD) and 139 age- and sex-matched healthy controls. In a case-control study conducted from 1991-2001 at the National Institute of Mental Health, Bethesda, MD, total cerebrum, cerebellum, gray and white matter for the four major lobes, and caudate nucleus volumes were measured by automated methods. On initial scan, patients with ADHD had significantly smaller brain volumes in all regions, amounting to a $3 \%$ decerease. Compared with controls, previously unmedicated ADHD children had significantly smaller total cerebral volumes, cerebellar volumes, and especially white matter volumes. In these unmedicated patients, white matter volumes were also smaller when compared with medicated ADHD children. Abnormal volumes persisted with age, except for caudate volumes which decreased in both patients and controls during adolescence. Male and female 